\title{
Water resources management
}

\author{
Gadea -Toledo, Ana Karina; Toruño, Pedro José; Castellón, Juan; \\ Geles-Roffe, Tatiana; Editor Academico Dr. Angel Sol Sanchez
}

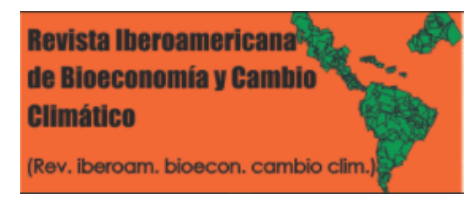

\author{
Ana Karina Gadea -Toledo \\ ana.gadea@post.unanleon.edu.ni \\ Universidad Nacional autónoma de Nicaragua \\ UNAN-León, CUR Jinotega, Nicaragua \\ (iD) Pedro José Toruño \\ Universidad Nacional Autónoma de Nicaragua, León, \\ Nicaragua \\ (iD) Juan Castellón \\ Universidad Nacional Autónoma de Nicaragua, León, \\ Nicaragua \\ Tatiana Geles-Roffe \\ Intituto de Geografía Tropical, Cuba \\ Editor Academico Dr. Angel Sol Sanchez \\ Colegio de postgraduados Mexico, Mexico
}

\author{
Revista Iberoamericana de Bioeconomía y Cambio \\ Climático \\ Universidad Nacional Autónoma de Nicaragua, León, Nicaragua \\ ISSN-e: 2410-7980 \\ Periodicidad: Semestral \\ vol. 2 , núm. 1,2016 \\ czuniga@ct.unanleon.edu.ni
}

Recepción: 09 Octubre 2015

Aprobación: 25 Abril 2016

URL: http://portal.amelica.org/ameli/journal/394/3941750010/

DOI: https://doi.org/10.5377/ribcc.v2i1.5703

Autor de correspondencia: ana.gadea@post.unanleon.edu.ni
Resumen: El presente trabajo se basa en resultados presentados por especialistas en recursos hídricos sobre experiencias de los sectores más vulnerables a sequía y aquellos que demandan agua de calidad tomando como referencia las zonas cafetaleras del norte de Nicaragua. Con base a una recopilación de información existente así como de entrevistas y talleres participativos utilizando plataformas de dialogo territorial con el objetivo de identificar las trayectorias más probables que podrían disminuir la vulnerabilidad de los productores de café (Coffea arabica) al cambio y variabilidad climática. En las conclusiones del estudio mencionamos que la tecnología de producción de café multiestrato con banano es un tema ampliamente estudiado utilizando un enfoque que aplica herramientas de toma de decisión para manejar la productividad del sistema - manejo del recurso luz, ciclos de nutrientes y optimización del banano como cultivo secundario, entre otros. Es necesario desarrollar un proceso participativo innovador para mejorar la gestión de los recursos hídricos en comunidades cafetaleras para incrementar su capacidad de adaptación y resiliencia frente al cambio climático (variabilidad de las lluvias y las sequías).

Palabras clave: Gestión de recursos hídricos, cambio climático, resiliencia, variabilidad climática.

Abstract: This work is based on results presented by specialists in water resources on experiences of the sectors most vulnerable to drought and those that demand quality water taking as a reference the coffee growing areas of northern Nicaragua. Based on a compilation of existing information as well as interviews and participatory workshops using territorial dialogue with the objective of identifying the most probable trajectories that could decrease the vulnerability of coffee (Coffea arabica) producers to climate change and variability. In the conclusions of the study, we mentioned that the technology for the production of multilayer coffee with bananas is a widely studied topic using an approach that applies decision-making tools to manage the productivity of the system - light resource management, nutrient cycles and banana optimization. as a secondary crop, among others. It is necessary to develop an innovative participatory process to improve the management of water resources in coffeegrowing communities to increase their capacity for adaptation and resilience to climate change (variability of rainfall and droughts). 
Keywords: Management of water resources, climate change, resilience, climate variability.

\section{INTRODUCCIÓN}

El cultivo de café (Coffea arabica) se encuentran ampliamente distribuido en Latinoamérica beneficiando a más de un millón de hogares produciendo ingresos, y otros servicios. A pesar de su gran aptitud climática, su productividad depende de la uniformidad en la disponibilidad de agua. En las últimas décadas, los registros y modelos climáticos han evidenciado un incremento de la temperatura promedio previendo que las temperaturas se incrementarán desde $2^{\circ} \mathrm{C}$ hasta el año 2030. A su vez, el incremento de temperaturas estará acompañado de una mayor variabilidad de lluvias con tempestades más frecuentes y sequías más fuertes yrecurrentes. Estos cambios serán un reto agronómico para los hogares cafetaleros adaptados a cultivar bajo regímenes climáticos más estables. El departamento de Jinotega produce el $65 \%$ del café a nivel nacional sin embargo esta producción ha venido disminuyendo con la amenaza generada por el cambio y variabilidad climática, En un contexto en el que se exigen cosechas de mayor uniformidad y calidad, las fincas productoras demandarán una seguridad en la cantidad y calidad de los recursos hídricos.Esta investigación contribuirá a que estas comunidades cafetaleras en alianza con diversos actores públicos, privados y de la sociedad civil gestionen los recursos hídricos para una mayor competitividad y resiliencia.

El café (Coffea arabica) se encuentran ampliamente distribuidos en Latinoamérica a menudo asociados bajo un enfoque agroforestal, (Staver et al.2010).El censo cafetalero del año 2001 refleja que del total nacional del área de producción del café que tiene como sombra el cultivo de banano 60,268 Ha están distribuidas al norte del país, usándola como una alternativa de sombra temporal y como autoconsumo familiar. En los últimos veinte años los bananos sembrados en los cafetales han pasado a formar parte del comercio en la finca convirtiéndose en una fuente importante de ingresos especialmente cuando los precios de café son bajos (Siles et al, 2010)

Nuestro estudio se centró en la revisión de la literatura sobre el tema de la gestión del recurso hídrico basado en la experiencia del proyecto Musaceas del grupo Biodiversity, caso Jinotega.

\section{REVISIÓN DE LA LiTERATURA}

\section{Caso Jinotega}

El departamento de Jinotega produce el $65 \%$ del café a nivel nacional esto gracias a las condiciones edafoclimaticas y biofísicas optimas de la zona sin; embargo A pesar de su gran aptitud climática, su productividad depende de la uniformidad en la disponibilidad de agua. Durante periodos de déficit de agua la emisión de hojas se detiene y el ciclo productivo se prolonga, mientras que durante periodos de saturación de los suelos la productividad puede verse amenazada (Fortescue et al. 2011).

En las últimas décadas, los registros y modelos climáticos evidencian un incremento paulatino de la temperatura promedio y concuerdan que las temperaturas se incrementarán desde $2^{\circ} \mathrm{C}$ hasta el año 2030. Estos incrementos acelerarán la tasa de emisión de hojas y una mayor demanda de agua. Según un estudio reciente de Bioversity (Calberto et al. 2015), el total de producción de hojas anuales aumentará hasta en un

\section{NotAS DE AUTOR}


$30 \%$, mientras la demanda de agua se incrementará de 15\% para el año 2070. A su vez, el incremento de las temperaturas estará acompañado de una mayor variabilidad de lluvias con tempestades más frecuentes y sequías más fuertes yrecurrentes.

Más allá de la amenaza generada por el cambio y variabilidad climática, la producción de café y las actividades en el campo están cada vez más sujetas a una reglamentación del Estado y a las exigencias del mercado, que ponen en riesgo la competitividad de los pequeños productores. En un contexto en el que se exigen cosechas de mayor uniformidad y calidad y en el que existe una demanda de agua de alta calidad para la post-cosecha y el manejo de las aguas residuales, las fincas productoras demandarán una seguridad en la cantidad y calidad de los recursos hídricos. Además de las pequeñas fincas, otras actividades agropecuarias, agroindustriales y urbanas también buscarán asegurar su acceso a los recursos hídricos en sus estrategias competitivas. Estas múltiples demandas futuras sobre el recurso agua como elemento clave en la productividad, la competitividad y la calidad de la vida humana constituyen el contexto de esta propuesta.

En esta investigación retomaremos el enfoque socio-ecológico/productivo no solamente para reconciliar producción y servicios ecosistémicos, sino también para identificar los posibles caminos que pueden conducir a la resiliencia de la finca y el territorio frente a los cambios y variabilidad climática. La resiliencia involucra la capacidad del sistema socio-ecológico/productivo para responder a la variabilidad en el clima y muchos otros factores. La capacidad de respuesta se plantea en función del tipo y la escala del disturbio, choque o variabilidad. Se puede plantear respuestas de diferentes tipos tales como manejos para evitar los efectos, para tolerarlos y para acelerar la posterior recuperación (Martin-Breen y Anderies 2011; Altamirano, 2009; Olsson, 2004). A través del tiempo por medio de un aprendizaje adaptativo, los actores e instituciones del sistema socio- ecológico/productivo ajustarán sus principios, reglas y prácticas de manejo (Smith y Stirling 2010).

Las cuatro comunidades donde se desarrollara corresponden a la Central de Cooperativas de Servicios Múltiples "Productores de Café Especiales", se fundó en el año 2014 con la participacion inicial de 330 socios de los cuales 85 están conformado por mujeres. A la fecha todos los socios se mantienen activo como miembros de la cooperativa. se caracteriza por el arduo empeño de trabajar a la par de sus socios y entidades estatales y no gubernamentales, así mismo han trabajado con instituciones de educación superior del país (Universidad Nacional Agraria, UNAN-FAREM, Matagalpa y hoy día con la prestigiosa Universidad Nacional Autónoma de Nicaragua (UNAN-León). Este acercamiento con las instituciones que desarrollan investigaciones ha permitido a la cooperativa, encontrar modelos productivos sostenibles que han venido permitiendo a mejoramiento de los niveles de vida de los socios y familias de escaso recursos.

\section{El café y el cambio climático}

Según Siles et al (2011) :Los cambios climáticos que vienen ocurriendo al nivel global están ocasionando efectos adversos sobre los cultivos agrícolas y el cafeto no es la excepción. Estas variaciones del clima se manifiestan en bajas en la producción, rendimiento y calidad de los productos. Como consecuencia se reflejará en la escasez del producto, disminución de los abastos y un posible aumento en los precios a los consumidores.

En el caso del café ya algunos países están enfrentando el reto de este cambio climático que se ha manifestado principalmente en un incremento de la temperatura. Esta condición se ha expandido a zonas de alta elevación donde crecen y se producen los cafés de calidad superior que son los mejor cotizados en el mercado. La situación forzará a los caficultores a adaptarse a las nuevas condiciones del tiempo adoptando prácticas que minimicen los efectos del aumento en temperatura y otros componentes del clima. También puede movilizar a los productores hacia tierras más altas en búsqueda de lugares que provean mejores condiciones ecológicas al crecimiento, desarrollo y fructificación del cafeto. Esto a su vez puede ocasionar la invasión de nuevos territorios no cultivados antes con el impacto que pueda tener en la transformación de 
bosques u otros sistemas a tierras de cultivo. Hay que mencionar además, como algo positivo que ese aumento en la temperatura puede ayudar a transformar regiones muy frías para el cultivo del cafeto en áreas propias para la producción de café.

El problema será más crítico para productores en áreas marginales de baja o mediana altitud donde ocasionará dificultades mayores.. Las plantaciones de café que sobrevivan el impacto del calentamiento global les aumentará las posibilidades de susceptibilidad al ataque de nuevas plagas que surjan bajo esas condiciones.

De otra parte, cambios en los patrones de lluvia (sequía o aumento) así como fuertes vientos son ejemplos también de cómo el cambio climático global puede dañar los cultivos reduciendo su producción y rendimientos.

\section{CECOSPROCAES-RL Café, Jinotega, Nicaragua:}

La Central de Cooperativas de Servicios Múltiples "Productores de Café Especiales”, se fundó en el año 2014 con la participación inicial de 330 socios de los cuales 85 están conformado por mujeres. A la fecha todos los socios se mantienen activo como miembros de la cooperativa. se caracteriza por el arduo empeño de trabajar a la par de nuestros socios y entidades estatales y no gubernamentales, así mismo han trabajado con instituciones de educación superior del país (Universidad Nacional Agraria, UNAN-FAREM, Matagalpa y hoy día con la prestigiosa Universidad Nacional Autónoma de Nicaragua (UNAN-León). Este acercamiento con las instituciones que desarrollan investigaciones ha permitido a la cooperativa, encontrar modelos productivos sostenibles que han venido permitiendo a mejoramiento de los niveles de vida de nuestros socios y familias de escaso recursos.

La Central de Cooperativas de Servicios Múltiples "Productores de Café Especiales", se fundó en el año 2014 con la participación inicial de 330 socios de los cuales 85 están conformado por mujeres. A la fecha todos los socios se mantienen activo como miembros de la cooperativa. se caracteriza por el arduo empeño de trabajar a la par de nuestros socios y entidades estatales y no gubernamentales, así mismo han trabajado con instituciones de educación superior del país (Universidad Nacional Agraria, UNAN-FAREM, Matagalpa y hoy día con la prestigiosa Universidad Nacional Autónoma de Nicaragua (UNAN-León). Este acercamiento con las instituciones que desarrollan investigaciones ha permitido a la cooperativa, encontrar modelos productivos sostenibles que han venido permitiendo a mejoramiento de los niveles de vida de nuestros socios y familias de escaso recursos.

\section{Variabilidad climática $(V C)$ :}

\section{Variabilidad}

Detrás del promedio en precipitación y temperatura existe la variabilidad climática que no es captada por los modelos y proyecciones. La variabilidad incluye eventos extremos e incertidumbre, que fluctúan cada año. Por esta razón se debe considerar el pasado para entender los tipos de eventos climáticos que históricamente han tenido suceso en las localidades seleccionadas.

Datos climáticos: CRU

Los datos utilizados para estimar la variabilidad histórica anual se obtuvieron de CRU (Climate Research Unit) (series de tiempo). Los datos contienen variaciones mes a mes en el clima durante el último siglo. Estos se calculan en grillas de alta resolución ( $0.5 \times 0.5$ grados), que se basan en un archivo de temperaturas medias mensuales proporcionados por más de 4000 estaciones meteorológicas distribuidas por todo el mundo. Los datos permiten que las variaciones en el clima sean estudiadas, e incluye variables tales como la cobertura de nubes, rango de temperaturas diurnas, frecuencia de días con heladas, precipitación, temperatura media 
diaria, la temperatura máxima diaria promediada mensualmente, la presión de vapor de agua y la frecuencia de días húmedos.

Para determinar la variabilidad interanual se tomaron datos mensuales para el periodo entre 1950 - 2009 y se usó el promedio entre 1960 - 1990 como referencia para ser comparados todos los años en función de las temperaturas y precipitaciones, para estimar si los valores están por encima o por debajo del promedio. Los años más cercanos al punto 0 - 0 significan años con valores exactos al promedio. Los años más alejados a este punto varían del promedio por temperatura o precipitación o por los dos factores. Años que se encuentran dentro del rectángulo naranja se consideran años con incrementos en valores promedios, años que se encuentran fuera del rectángulo naranja y dentro del rojo se consideran años con variabilidad moderada; finalmente años por fuera del rectángulo rojo se consideran años con variabilidad extrema.(Figura 1)

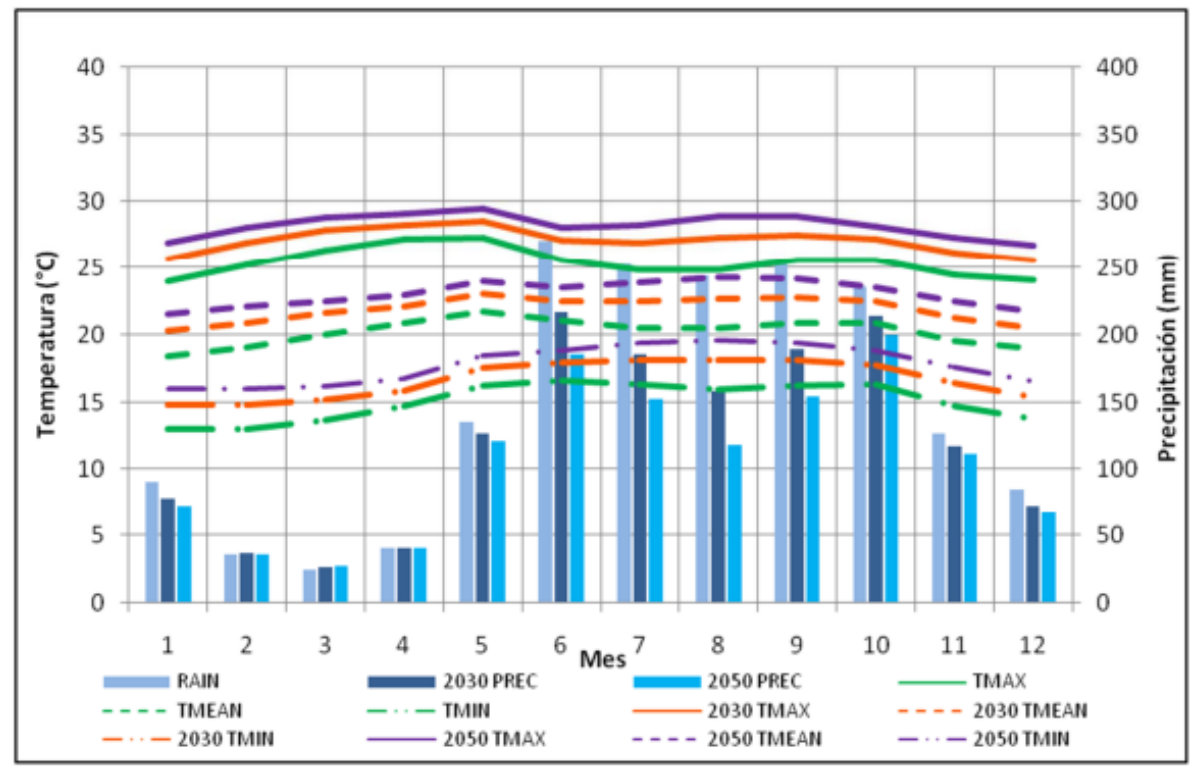

FIGURA 1.

Temperatura y precipitación media mensual usando valores de 20 GCMs bajo el escenario A2 para comunidad America - Nicaragua. Elaboración propia

Para elaborar las proyecciones se utilizaron los datos obtenidos del portal CCAFS1. Se usó el escenario A2, una resolución de 2.5 minutes $(\sim 5 \mathrm{~km})$, y se promediaron los valores esperados de 20 modelos de circulación general (GCM).

Las proyecciones para las variables de temperatura y precipitación se realizaron para las áreas seleccionadas (Tabla 1).

TABLA 1.

Localidades seleccionadas

\begin{tabular}{llcc}
\hline Localidad & País & $\begin{array}{c}\text { Temperatura } \\
\text { media }\left({ }^{\circ} \mathbf{C}\right)\end{array}$ & $\begin{array}{c}\text { Precipitación anual } \\
(\mathbf{m m})\end{array}$ \\
\hline Comunidad América & Nicaragua & 20 & 1770 \\
\hline
\end{tabular}




\section{Gestión y manejo integrado de cuencas}

Unidad territorial en la cual el agua que cae por precipitación se reúne y escurre en un punto común fluyendo toda al mismo río, lago o mar. Esta unidad natural definida por la existencia de la divisoria de aguas en un territorio dado, es también conocida como "parteaguas". Las cuencas se pueden delimitar en: subcuencas o microcuencas o cuencas de orden inferior y están formadas por un conjunto de elementos que se interrelacionan. Los más importantes son: el agua, el bosque y el suelo (Campos,2000).

Cuenca Hidrográfica

Unidad territorial en la cual el agua que cae por precipitación se reúne y escurre en un punto común fluyendo toda al mismo río, lago o mar. Esta unidad natural definida por la existencia de la divisoria de aguas en un territorio dado, es también conocida como "parteaguas". Las cuencas se pueden delimitar en: subcuencas o microcuencas o cuencas de orden inferior y están formadas por un conjunto de elementos que se interrelacionan. Los más importantes son: el agua, el bosque y el suelo (Campos,2000).

Cuenca Hidrológica

Son unidades morfológicas integrales que incluyen el concepto de cuenca hidrográfica y toda la estructura hidrogeológica subterránea del acuífero. Para llevar a cabo el Manejo integrado de Cuencas hay que partir de un diagnóstico. La importancia de trabajar en las cuencas es que se debe priorizar la conservación de suelos y agua, el reciclaje de suelos y de materia orgánica y la reforestación con especies nativas como estrategias claves para mejorar las condiciones básicas de producción (Campos 2000).

En la Cuenca se puede medir el balance hídrico, se puede saber cuanta agua entra, se evapora, se transpira, se escurre se infiltra y finalmente cuanto sale de ella al mar, lago o río, pero también se pueden analizar todas las actividades productivas que se desempeñan a su alrededor, el agua es el indicador de cómo Estudio de la microcuenca y diseño de Plan de Accion Participativo para potenciar la disponibiliad y calidad de agua. La cuenca nos sirve para hacer un análisis integral ambiental, geofisico y socioeconomico (Etienne, 2013).

Por lo tanto, el concepto Gestión Integral implica el desarrollo de capacidades locales que faciliten la participación. El fin de los planes de gestión y manejo integral es el conducir al desarrollo de la cuenca a partir de un uso sustentable de los recursos naturales. Un aspecto muy importante para este logro, radica en la estrategia de capacitación agrícola, principalmente la formación integral de un conjunto de promotores y líderes de la comunidad por su comunidad. Para ello, hay que apoyarse en la comunidad, en las organizaciones campesinas existentes en la cuenca, reforzado con el de organizaciones, permitiendo con ello movilizar la fuerza de trabajo necesaria para las faenas conservacionistas, a través de trabajos colectivos, ya que las familias tradicionalmente buscan la cooperación de vecinos, parientes y amigos para las faenas principales (Altamirano ,2009).

En Nicaragua, actualmente existen condiciones legales que permiten la participación ciudadana para la búsqueda de alternativas de problemas locales especialmente las relacionadas con la conservación y uso adecuado de los recursos hídricos siendo una prioridad para el desarrollo de la nación y el bienestar de la población.

La Constitución Política de Nicaragua en su Artículo No. 7, La Ley de Municipios en su Artículo No. 36 y La Ley de Participación Ciudadana garantiza un marco legal para la participación comunitaria en la gestión de los proyectos de desarrollo local, con la finalidad de incidir y participar en la toma de decisiones para lograr el desarrollo humano sostenible.

Diálogo territorial multiactores para la formulación participativa de un plan de acciones para el manejo de los recursos hídricos en las comunidades cafetaleras.

Cada plataforma de diálogo territorial participativa permitirá que los actores de cada comunidad cafetaleras exploren diferentes escenarios, evalúen el efecto de sus acciones sobre la disponibilidad de los recursos hídricos y propongan diferentes acciones, soluciones, estrategias e innovaciones para disminuir su vulnerabilidad a la variabilidad climática. Durante estos espacios de prospección, los actores podrán analizar 
los efectos de la variabilidad climática sobre la productividad, la producción, los costos y la calidad del producto, variables que afectan la comercialización. En función del análisis de la situación actual y de los escenarios futuros, las comunidades y sus organizaciones se podrán organizar en comisiones para explorar alternativas y luego definir un plan de acciones de gestión de los recursos hídricos para la producción y comercialización agricola a corto, mediano y largo plazo, como estrategia de adaptacion al cambio climatico. Se esperan identificar acciones que se puedan aprender de inmediato sin mayor apoyo externo a la comunidad así como tambien acciones que requieran nuevas alianzas y que sean más exigentes en recursos.

\section{Modelamiento participativo}

La modelización participativa es un método que le permite a un grupo de participantes desarrollar y construir un "modelo". Este modelo corresponde a la representación de una situación dada, de un problema o de un sistema. En función de la pregunta tratada diferentes tipos de modelos se pueden desarrollar: relación entre actores, dinámica de los recursos, relación entre actores/recursos y sus procesos de transformación, entre otros. La fuerza que posee este método consiste en reunir a los participantes alrededor de un objeto común, el cual fue construido por el conjunto de actores. Este objeto (el modelo) sirve posteriormente para definir objetivos, alternativas, estructurar preguntas o problemas(ComMod,2011).

\section{Juego de Roles}

El juego de roles se define como la puesta en escena de una situación problema en la que participan personas que juegan un rol específico o establecido. Dentro de un proceso ComMod, el juego de roles conduce a los participantes a encontrarse en una situación en la que deben discutir y tomar decisiones colectivas e individuales, permitiendo que se busquen soluciones conjuntas a los problemas.

Los juegos de roles permiten que un grupo de actores tengan una representación conjunta de un problema, permitiendo que se genere un espacio de discusión sin tensión entre diferentes grupos de interés así como su amplia participación. En efecto, se ha observado que los juegos de roles crean los espacios para que se discutan temas sensibles porque permiten, en la reproducción de una situación problema que ocurre en la realidad, que los jugadores tomen una distancia con el mundo real y dialoguen sobre este tema(ComMod,2011).

\section{Conclusiones}

La tecnología de producción de café multiestrato con banano es un tema ampliamente estudiado utilizando un enfoque que aplica herramientas de toma de decisión para manejar la productividad del sistema - manejo del recurso luz, ciclos de nutrientes y optimización del banano como cultivo secundario, entre otros.

Es necesario desarrollar un proceso participativo innovador para mejorar la gestión de los recursos hídricos en comunidades cafetaleras para incrementar su capacidad de adaptación y resiliencia frente al cambio climático (variabilidad de las lluvias y las sequías).

Que las comunidades involucradas, identifiquen conocimientos, fuentes de recursos y socios específicos para emprender acciones a corto y mediano plazo en los aspectos productivos, políticos y de investigación vinculadas a la resiliencia y productividad/ingreso en el manejo adaptativo del agua como servicios ecosistémicos de su comunidad. 


\section{ReFERENCIAS}

Altamirano, M. (2009). Distribucion de la contaminación natural por Arsénico en las aguas subterráneas de la subcuenca suroeste del Valle de Sébaco. Maestría thesis, Universidad Nacional Autónoma de Nicaragua

Calberto, G., Staver, C., \& Siles, P. (2015). An assessment of global banana production and suitability under climate change scenarios. Climate change and food systems: global assessments and implications for food security and trade. Rome: Food Agriculture Organization of the United Nations (FAO).

Campos Ortiz, M. (2000). Escenarios climáticos y socioeconómicos de Nicaragua para el siglo XXI. Ministerio del Ambiente y los Recursos Naturales, Managua (Nicaragua).

Étienne, M. (Ed.). (2013). Companion modelling: a participatory approach to support sustainable development. Springer Science \& Business Media.

Fortescue, J. A., Turner, D. W., \& Romero, R. (2011). Evidence that banana (Musa spp.), a tropical monocotyledon, has a facultative long-day response to photoperiod. Functional Plant Biology, 38(11), 867-878

Martin-Breen, P., \& Anderies, J. M. (2011). Resilience: a literature review. The Rockefeller Foundation. New York.

Olsson, P., Folke, C., \& Berkes, F. (2004). Adaptive comanagement for building resilience in social-ecological systems. Environmental management, 34(1), 75-90.

Siles, P., Bustamante, O., Deras, M., Matute, O., Aguilar, C., Rojas, J., ... \& Staver, C. (2010). Bananos en cafetales con árboles en América Latina: estrategias preliminares para mejorar su productividad, rentabilidad y sostenibilidad. XIX Reunión Internacional ACORBAT. Medellin, CO.

Smith, A., \& Stirling, A. (2010). The politics of social-ecological resilience and sustainable socio-technical transitions. Ecology and society, 15(1).

Staver, C., O. Bustamante, P. Siles, P. Talavera, D. Brown, H. Garming, L. Pocasangre, G. Soto. 2010. Bananas and agrobiodiversity in Mesoamerican agricultural landscapes. 6th Henry A. Wallace Conference. Agrbiodiversity in Mesoamerica - from Genes to Landscapes. Turrialba, Costa Rica. 20-24 September.

Siles, P., Aguilar, C., Quinde, K., Castellón, J., Somarriba, F., Tapia, A., ... \& Bustamante, O. (2011, October). Intercropping bananas with coffee and trees: prototyping agroecological intensification by farmers and scientists. In VII International Symposium on Banana: ISHS-ProMusa Symposium on Bananas and Plantains: Towards Sustainable Global Production 986 (pp. 79-86). 